\title{
Hardware Demonstrator Of The Phase-II ATLAS MDT Trigger Processor
}

\section{Cieri* on behalf of the ATLAS Collaboration}

Max-Planck-Institut für Physik, Föhringer Ring 6, 80805 Munich, Germany

E-mail: davide.cieriecern.ch

The first level muon trigger of the ATLAS experiment will be upgraded to operate at the HighLuminosity LHC. The selectivity of the current system is limited by the moderate spatial resolution of RPC and TGC. The MDT chambers currently used for precision tracking will be therefore included to improve the momentum resolution and the redundancy.

In the upgraded muon trigger system, the MDT trigger processors will receive MDT hits from the detectors and match them to the trigger candidates (seeds) from the RPC and TGC trigger systems. These seeds provide a Region-of-Interest (RoI) and the bunch-crossing timing which is used for calculating the MDT drift time. MDT hits matched to the RoI are then used by the MDT trigger algorithm to improve the momentum resolution, by forming track segments and joining them together for momentum determination.

A hardware demonstrator of the MDT trigger processor, based on a common ATCA platform known as "APOLLO", is currently under production. It consists of two separate modules called the "Command Module" and "Service Module", and it is based on FPGA technology.

A description of the algorithms for the MDT track reconstruction is presented. The achieved trigger performance allows to reconstruct muon tracks with a momentum resolution of about $6 \%$ and a trigger efficiency above $95 \%$ for muons with a transverse momentum of $20 \mathrm{GeV}$.

Topical Workshop on Electronics for Particle Physics (TWEPP2019)

2-6 September 2019

Santiago de Compostela, Spain

* Speaker. 


\section{Introduction}

The ATLAS [1] muon spectrometer consists of a combination of Resistive Plate Chamber (RPC) and Thin Gap Chamber (TGC) for triggering, and Monitored Drift Tube (MDT) for precise momentum measurements.

The MDT Trigger Processor (MDTTP) will be a major component of the Phase-II upgrade of the ATLAS muon trigger and readout system [2]. The precision coordinates measured by the MDT chambers will be used to improve the momentum measurements of muon candidate tracks in the hardware-based Level-0 (L0) muon trigger. This will guarantee a high trigger efficiency while maintaining a low $\mathrm{L} 0$ trigger rate.

For this purpose, the MDT front-end electronics will be replaced to allow a trigger-less transmission of the MDT hits to off-detector [3]. The Barrel and Endcap Sector Logic (SL) boards will process trigger candidates from the RPC, TGC and New Small Well (NSW) data, which will be used as seeds of the MDTTP track reconstruction algorithm. The MDTTP identifies the MDT hits compatible with the Region-of-Interest (RoI) and bunch-crossing timing provided by the SL (Hit Extraction). The extracted hit information is therefore exploited to reconstruct muon track segments within each MDT chamber. Finally, the segment parameters are combined to determine the muon transverse momentum $p_{\text {T }}$ precisely.

The MDTTP will also be responsible for the transmission of the MDT hits to FELIX [4], upon the receiving of an L0-accept signal, and the monitoring and configuration of the MDT chamber front-end electronics.

The MDTTP will be physically implemented as an ATCA blade and will process data of one sector of the muon spectrometer. Since the muon spectrometer is segmented into 64 sectors, 64 MDTTP boards are needed to operate the system.

\section{The MDT Trigger Processor Hardware Demonstrator}

A hardware demonstrator for the MDTTP is currently under preparation. It is based on a common ATCA platform known as "APOLLO" [5]. The blade consists of two separate modules: the "Service Module" (SM) and "Command Module" (CM) (Fig. 1a).

The SM is not application-specific and provides basic services, including module management, power conditioning, ethernet and firmware management. The CM, on the other hand, is applicationspecific and handles all the data processing and external communications. It provides one Xilinx Kintex Ultrascale+ FPGA (XCKU15P) and a Xilinx Zynq Ultrascale+ SoC (XCZU11EG) [6]. Hit extraction and segment finding tasks are operated independently in the two devices. The Kintex FPGA is responsible for two chambers, and the Zynq FPGA is for the remaining chamber in the same sector. The Kintex also provides the interface to the SL board and to FELIX, while the track fitting is implemented into the Zynq.

\section{The MDT Segment Finder}

The MDTTP reconstructs muon tracks by combining the segment information from each MDT chamber. Within a single chamber, muons are assumed to have a straight-line trajectory for the 
momenta relevant to the trigger. Two algorithms are currently investigated for the segment finding on FPGA. An option consists of using a two-dimensional Legendre Transform array, scanning over the segment position and angle around the SL seed, where segment candidates are given by the most populated bin in the array [7]. Another proposal, known as Compact Segment Finder (CSF), is based on a one-dimensional scan over the segment position, using the SL seed for the direction. The selected MDT hits are finally utilised for a $\chi^{2}$ fit, returning the precise segment parameters [8] and a result of the fitting quality, which is not available for the Legendre algorithm.

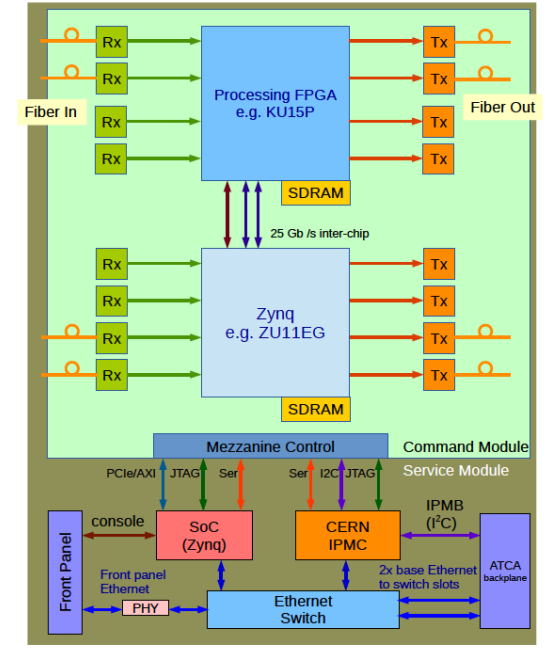

(a) Block diagram of the MDTTP blade showing ing algorithms, as implemented in a Kintex Ultrascale+ the major components on the SM and the CM. xcku15p FPGA [6].

An alternative design option under consideration exploits the use of Associative Memory ASICs to identify muon segments using precomputed track segment patterns. This option requires, however, different hardware architecture and is not supported by the MDTTP ATLAS demonstrator.

Both the Legendre and the CSF options have been validated using simulated samples with the official ATLAS Phase-II geometry, showing similar performance in terms of segment finding efficiency and the position and angle resolution of the track segments. They have also been successfully implemented in HDL, requiring a low resource usage (see Table 1b), allowing the implementation of at least nine segment finders per MDTTP blade, necessary to reconstruct up to three muons in a single trigger sector. Both approaches present a low latency, determining the segment parameters in less than $200 \mathrm{~ns}$ with an FPGA clock frequency of $360 \mathrm{MHz}$.

\section{The MDT Momentum Determination}

The muon track segments reconstructed in the MDT are combined in the Zynq SoC to determine the refined muon transverse momentum. Depending on the number of reconstructed segments, two different variables can be used to compute $p_{\mathrm{T}}$.

If three segments of sufficient quality are available, it is possible to calculate the sagitta $s$, defined in the barrel as the distance in the bending plane of the segment in the middle chamber 
from the straight line connecting the segments in the inner and the outer chambers. In the endcaps a pseudo-sagitta definition can be used, which is the distance in the bending plane of the segment in the inner chamber from the line connecting the middle and outer chambers (Fig. 2a).

If only two segments of sufficient qualities have been reconstructed, the angle $\Delta \beta$ between the direction of the two segments in the bending plane can be computed (Fig. 2b).

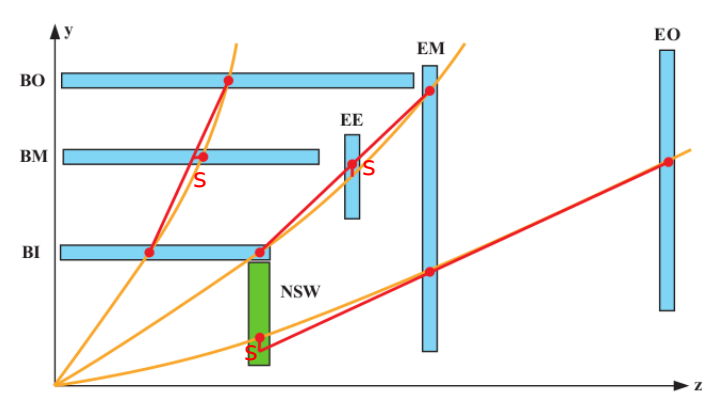

(a)

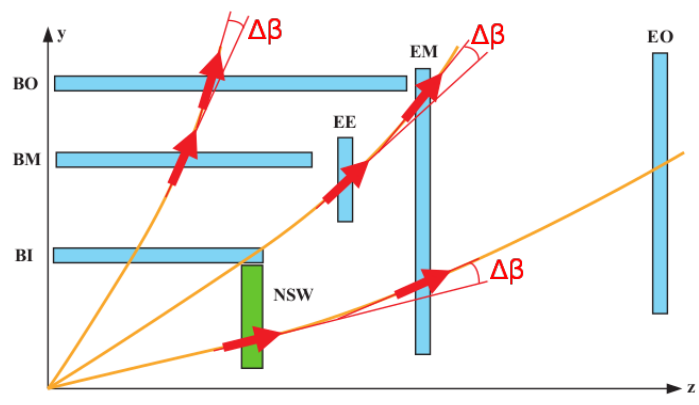

(b)

Figure 2: Schematic view of the concept of the sagitta of three segments (2a) and of the polar angle difference $\Delta \beta$ between two segments (2b) [2].

Finally, the transverse momentum can be expressed as a function of $s(\Delta \beta), \phi$ and $\eta$,

$$
\begin{aligned}
& p_{\mathrm{T}}=\sum_{i=0}^{2} \frac{a_{i}}{s^{i}}+\sum_{j=0}^{2} b_{j} \cdot \phi^{j}+\sum_{k=0}^{1} c_{k} \cdot \eta^{k}, \\
& p_{\mathrm{T}}=\sum_{i=0}^{2} \frac{d_{i}}{\Delta \beta^{i}}+\sum_{j=0}^{2} e_{j} \cdot \phi^{j}+\sum_{k=0}^{1} f_{k} \cdot \eta^{k},
\end{aligned}
$$

where $a_{i}, b_{j}, c_{k}, d_{i}, e_{j}$ and $f_{k}$ are parametrisation constants, calculated for each possible chamber combination.

\section{LOMDT Trigger Performance}

The LOMDT Trigger performance has been estimated using simulated data samples of single muon signals with the latest ATLAS Phase-II muon spectrometer geometry with no pile-up.

Muon tracks reconstructed using offline segment information with the sagitta or $\Delta \beta$ method have a good momentum resolution (Fig. 3a), about $6 \%$ for muons with $p_{\mathrm{T}}$ of $20 \mathrm{GeV}$ [2]. The trigger efficiency (Fig. 3b) has been measured using muon track segments found by the CSF algorithm. The MDT trigger provides an improved selectivity for the muons with $p_{\mathrm{T}}$ around the $20 \mathrm{GeV}$ threshold, keeping a high-plateau efficiency. The inclusion of the MDT trigger reduces the L0 muon trigger rate by about $55 \%$.

\section{Conclusions}

A hardware demonstrator for the MDT Trigger Processor based on the "APOLLO" framework is under production. Two FPGA-based algorithms for the segment finding task are considered. 


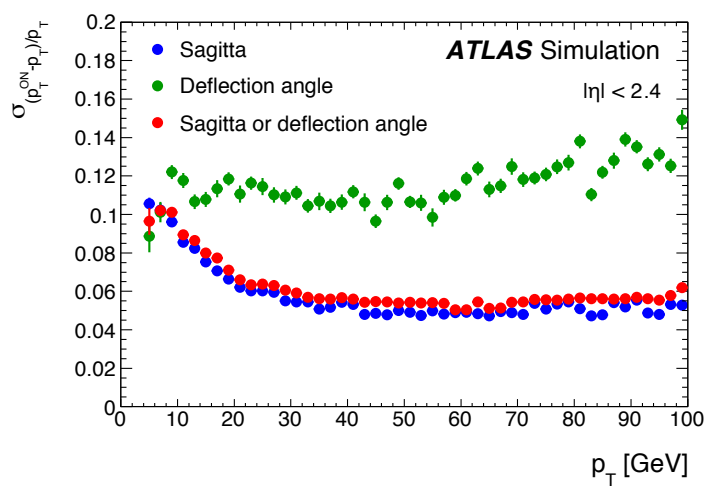

(a)

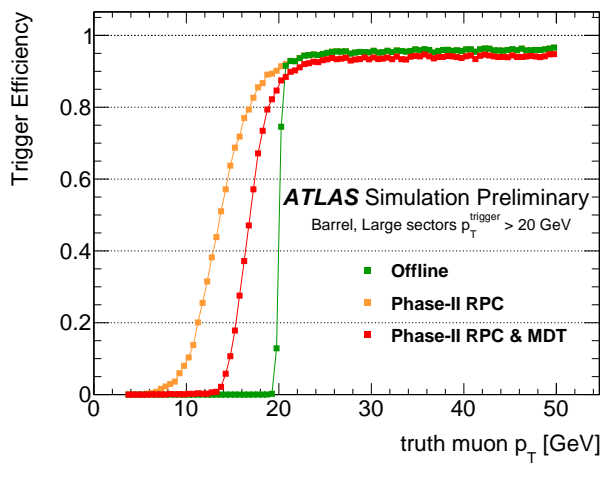

(b)

Figure 3: $p_{\mathrm{T}}$ resolution of the LOMDT muon tracks as a function of the offline muon $p_{\mathrm{T}}$ (3a) [2]. L0MDT trigger efficiency for a trigger threshold of $20 \mathrm{GeV}$ as a function of the truth muon $p_{\mathrm{T}}$ for the large sectors in the barrel, compared with a standalone RPC trigger and a full-offline reconstruction (3b) [9].

They both allow the reconstruction of up to three muons in the same trigger sector within the assigned latency.

Muon track fitting performance has been estimated with a Monte Carlo sample with the ATLAS Phase-II geometry. A better momentum resolution and a sharper turn-on efficiency curve are obtained by including the MDT information to the L0 muon trigger. A significant rate reduction of about $55 \%$ is expected for the single muon trigger with a $p_{\mathrm{T}}$ threshold of $20 \mathrm{GeV}$.

\section{References}

[1] ATLAS Collaboration, The ATLAS Experiment at the CERN Large Hadron Collider, 2008 JINST 3 S08004.

[2] ATLAS Collaboration, Technical Design Report for the Phase-II Upgrade of the ATLAS TDAQ System, ATLAS-TDR-029, https://cds.cern.ch/record/2285584.

[3] ATLAS Collaboration, Technical Design Report for the Phase-II Upgrade of the ATLAS Muon Spectrometer, ATLAS-TDR-026, https://cds.cern.ch/record/2285580.

[4] ATLAS Collaboration, FELIX: the New Detector Interface for the ATLAS Experiment, ATL-DAQ-PROC-2018-003, https://cds.cern.ch/record/2624299.

[5] E. Hazen, The APOLLO ATCA Platform, proceeding at TWEPP2019.

[6] Xilinx Co., UltraScale Architecture and Product Data Sheet: Overview.

[7] ATLAS Collaboration, Fast track segment finding in the Monitored Drift Tubes of the ATLAS Muon Spectrometer using a Legendre transform algorithm, ATL-DAQ-PROC-2018-008.

[8] D. Cieri et al., Hardware demonstrator of a compact first-level muon track trigger for future hadron collider experiments, JINST 14 (2019) 02, P02027.

[9] ATLAS Collaboration, LO Muon Trigger Public Result, https://twiki.cern.ch/twiki/bin/view/AtlasPublic/L0MuonTriggerPublicReults. 